\title{
Comparison between a transurethral prostate split and transurethral prostate resection for benign prostatic hyperplasia treatment in a small prostate volume: a prospective controlled study
}

\author{
Bohan Wang", Shigeng Zhang", Chengfang Sun, Chuanjun Du, Gaofei He, Jiaming Wen \\ Department of Urology, The Second Affiliated Hospital, School of Medicine, Zhejiang University, Hangzhou, China \\ Contributions: Conception and design: B Wang, S Zhang, J Wen; (II) Administrative support: C Du; (III) Provision of study materials or patients: S \\ Zhang, J Wen; (IV) Collection and assembly of data: C Sun, G He; (V) Data analysis and interpretation: C Sun, J Wen; (VI) Manuscript writing: All \\ authors; (VII) Final approval of manuscript: All authors. \\ \#The authors contributed equally to this work. \\ Correspondence to: Jiaming Wen, MD, PhD. Department of Urology, The Second Affiliated Hospital, School of Medicine, Zhejiang University, No. \\ 88, Jiefang Road, Hangzhou, China. Email: wenjiaming@zju.edu.cn.
}

Background: Transurethral resection of the prostate (TURP) was considered the golden standard to treat benign prostatic hyperplasia (BPH) for decades. However, TURP was associated with low efficiency to alleviate the lower urinary tract symptoms (LUTS) and a significantly higher risk of bladder neck contracture (BNC) for patients with small-volume BPH. Our study aims to compare the therapeutic effect of a transurethral split of the prostate (TUSP) with TURP for patients with small-volume BPH $(<30 \mathrm{~mL}$ ).

Methods: In this study, 101 small-volume BPH patients were randomly divided into two groups (TUSP and TURP group). The patient's baseline characteristics and perioperative outcomes were recorded. The follow-up was done at six months, one year and two years after surgical treatment.

Results: No significant differences were observed between the two groups for the baseline characteristics, including age, prostate volume, prostate-specific antigen (PSA) level, concurrent disease, post-void residual (PVR), maximum urinary flow rate $\left(\mathrm{Q}_{\max }\right)$, international prostate symptoms score (IPSS), and quality of life (QoL) score. The operative time and hemoglobin decrease were significantly lower in the TUSP group compared to the TURP group. However, no significant differences were observed between both groups for catheterization time, postoperative hospital stay, and incidence of transurethral resection syndrome (TURS). However, of the late complications, the incidence of BNC in the TUSP group was significantly lower than the TURP group. No significant differences were found between both groups for other complications, including postoperative bleeding, micturition urgency, micturition frequency, micturition pain, urinary tract infection, recatheterization, transient incontinence, and continuous incontinence. Follow-up results showed that the IPSS of the TUSP group was significantly lower than the TURP group, while the $\mathrm{Q}_{\max }$ of the TUSP group was significantly higher than the TURP group.

Conclusions: This study shows that TUSP may be an efficient and safe treatment for small-volume BPH $(<30 \mathrm{~mL})$ with a lower incidence of postoperative BNC and better longtime clinical outcomes than TURP. It suggested that TUSP could be an ideal treatment choice for small-volume BPH.

Keywords: Benign prostatic hyperplasia (BPH); transurethral split of the prostate (TUSP); transurethral resection of the prostate (TURP); small-volume prostate; bladder neck contracture (BNC)

Submitted Jul 05, 2020. Accepted for publication Aug 10, 2020.

doi: 10.21037/atm-20-5462

View this article at: http://dx.doi.org/10.21037/atm-20-5462 


\section{Introduction}

Benign prostatic hyperplasia (BPH) is a common disease in aging men (1), characterized by abnormal enlargement of the prostate gland (2). This disease is associated with lower urinary tract symptoms (LUTS) and urinary obstruction, which always causes a decrease in quality of life (QoL) (3). Transurethral resection of the prostate (TURP) is regarded as the golden standard to treat $\mathrm{BPH}$ for decades, including the classic monopolar TURP and modified bipolar TURP. According to the European Association of Urology (EAU) 2019 guideline, TURP is recommended as a treatment choice for the small-volume BPH $(<30 \mathrm{~mL})$. From the guideline, TURP is also the first line/standard treatment for larger sized BPH (30-80 mL). However, conventional TURP has some operative complications, including blood loss, dilution hyponatremia, and hypervolemia. Moreover, some postoperative complications are observed for TURP, including bladder neck contracture (BNC), retrograde ejaculation, and urinary incontinence (4). BNC is partially due to the thermal injury followed by tissue resection (5). It is reported that the total incidence rate of BNC after TURP was 5.4\% (6), which usually occurred in 6 months after TURP.

Recently, many novel surgical treatments have been developed for treating BPH. Holmium laser enucleation of the prostate (HoLEP) is one of the most representative techniques. The EAU 2019 guideline recommend HoLEP as to the first-line therapeutic choice for large-size BPH $(>80 \mathrm{~mL})$ and a choice for moderate size BPH $(30-80 \mathrm{~mL})$. However, HoLEP is not recommended for the smallvolume $\mathrm{BPH}(<30 \mathrm{~mL})$ since the capsule of the prostate is not clear for the HoLEP procedure.

Some patients with small-volume BPH $(<30 \mathrm{~mL})$ showed severe LUTS, including a low maximum flow rate $\left(\mathrm{Q}_{\max }\right)$ and a high post-void residual (PVR) (7) rate, usually related to the bladder neck fibrosis and prostate fibrosis. With the EAU 2019 guideline, transurethral incision of the prostate (TUIP) serves as the first line/standard treatment for the small volume prostates $(<30 \mathrm{~mL})$ without a middle lobe. However, the treatment for small-volume BPH is a clinical challenge since it is reported that small-volume prostate is associated with a significantly higher risk of BNC after TURP (6). Thus, it is crucial to develop novel surgical treatments for small-volume BPH $(<30 \mathrm{~mL})$.

Transurethral dilation of the prostate (TUDP) was used to treat BPH patients in 1987 (8). However, due to the design defect and reduced, longtime clinical efficacy, TUDP was not extensively used for clinical BPH patients. Recently, the modified TUDP, which called the transurethral split of the prostate (TUSP), was developed (9). Different from the traditional TUDP procedure, TUSP is designed with a double-columnar balloon catheter. This catheter expands the prostatic urethra and bladder neck simultaneously. The hyperbaric water sac would spit the capsule of the prostate and relieve the bladder outlet obstruction (10). Several earlier studies showed the clinical safety and efficacy of TUSP. Huang et al. reported in 2015 that TUSP is an ideal choice for BPH patients with significant therapeutic effects and limited complications from the results of the clinical study and animal experiments (9). More recently, a further study indicated TUSP was a safe and long-term efficient treatment for $\mathrm{BPH}$ with minimal invasion, short operative time, few postoperative complications, and low recurrence rate (4), and this study involved $565 \mathrm{BPH}$ patients and follow-up for 38 to 99 months.

A recent study indicated that three major pathobiological processes, including proliferation and enlargement, smooth muscle contractility, and prostate fibrosis, could act alone or in combination to promote LUTS (11). For the smallvolume $\mathrm{BPH}(<30 \mathrm{~mL})$, prostate fibrosis and smooth muscle contractility rather than proliferation or enlargement are usually the main factors contributing to LUTS. We think TUSP involves expanding the prostatic urethra and bladder neck without tissue removal, which may provide a novel therapeutic option for small-volume BPH $(<30 \mathrm{~mL})$ and generate a lower incidence of BNC after surgery without thermal injury. To evaluate this possibility, we compare the therapeutic effect of TUSP with TURP for small-volume $\mathrm{BPH}$ patients to explore a possible new method to treat small-volume BPH $(<30 \mathrm{~mL})$. In this prospective study, we find that TUSP may be an efficient and safe treatment for small-volume BPH $(<30 \mathrm{~mL})$ with a lower incidence of postoperative BNC and better longtime clinical outcomes than TURP. We present the following article in accordance with the CONSORT reporting checklist (available at http://dx.doi.org/10.21037/atm-20-5462).

\section{Methods}

\section{Patients}

All procedures performed in this study involving human participants were in accordance with the Declaration of Helsinki (as revised in 2013). The Ethics Committee approved this study of the Second Affiliated Hospital of 


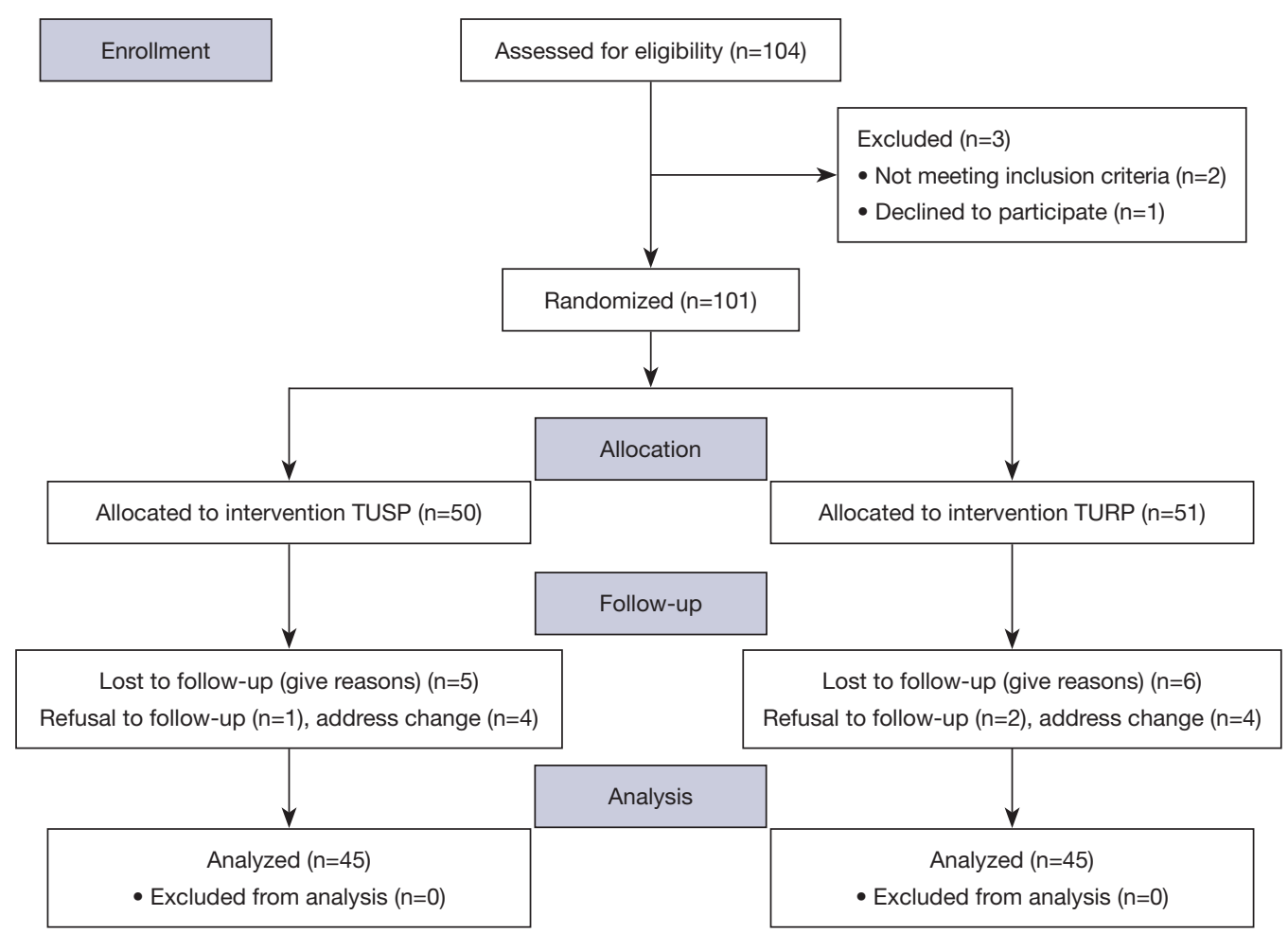

Figure 1 CONSORT, 2010 Flow Diagram. TUSP, transurethral split of the prostate.

Zhejiang University School of Medicine. It was registered in the Clinical Trial Registry of China with the trial number ChiCTR1900021803. Given the prospective controlled study design, a written informed consent was obtained from all the patients. An agreement was received for all patients before the enrollment. This work was followed by the Consolidated Standards of Reporting Trials (CONSORT) guidelines. A total of 101 patients (50 for the TUSP group and 51 for the TURP group) who underwent prostate surgery between May 2016 and January 2018 in our department were enrolled in this study (Figure 1). Height $\times$ length $\times$ width $\times \pi / 6$ calculated the patients' prostate volume through trans-rectal ultrasound before surgery in our study. IPSS and QoL score were obtained through the corresponding questionnaires. $\mathrm{Q}_{\max }$ was obtained through the urodynamic examination and PVR was obtained through the bladder ultrasound. Inclusion criteria for this study include: (I) the BPH patients with surgical indication; (II) Qmax $<12 \mathrm{~mL} / \mathrm{s}$; (III) prostate volume $<30 \mathrm{~mL}$; (IV) QoL score $>3$; (V) international prostate symptoms score (IPSS) $>21$; (VI) bladder trabeculation or diverticulum under cystoscopy; (VII) medication of $\alpha$-adrenergic blockers for at least 3 months. Patients with acute prostatitis, urethritis, neurogenic bladder, abnormal prostate-specific antigen (PSA) level, and urethral injury history were excluded from this study. Computer-generated allocation sequence randomized patients to TUSP or TURP group with allocation ratio $1: 1$ before surgery and their characteristics were shown in Table 1.

\section{Operative procedure}

Figure 2 shows the structure of the double-columnar balloon catheter, which was designed and manufactured by the UNIKITON company in China (The official website about the device is: http://www.unikiton.com/html/Prd/a/238. html). The procedure of TUSP was described before (4). Briefly, the procedures were performed under general anesthesia or spinal anesthesia in a lithotomy position. The columnar catheter was first put into the bladder through the urethra and then pulled out gradually until positioning protrusion passing though the membranous urethra. When pulling out the catheter, our finger was put into the rectum to watch the position of positioning protrusion. The positioning protrusion disappeared in the rectum and instantly appeared in subcutaneous perineum, which was the 
Table 1 Baseline patients' characteristics

\begin{tabular}{lccc}
\hline Parameters & TUSP $(\mathrm{n}=50)$ & TURP $(\mathrm{n}=51)$ & $\mathrm{P}$ value \\
\hline Age $(\mathrm{yr})$. & $70.3 \pm 7.3$ & $71.2 \pm 7.8$ & 0.550 \\
Prostate volume $(\mathrm{mL})$ & $25.5 \pm 1.5$ & $25.9 \pm 1.6$ & 0.198 \\
Qmax $(\mathrm{mL} / \mathrm{s})$ & $7.8 \pm 1.8$ & $7.6 \pm 1.5$ & 0.545 \\
PVR $(\mathrm{mL})$ & $50.3 \pm 27.9$ & $48.5 \pm 21.3$ & 0.716 \\
IPSS & $23.6 \pm 2.3$ & $23.9 \pm 2.1$ & 0.495 \\
QoL & $4.3 \pm 0.4$ & $4.4 \pm 0.5$ & 0.270 \\
PSA & $1.4 \pm 1.3$ & $1.5 \pm 1.2$ & 0.688 \\
Concurrent diseases, $(\mathrm{n})$ & & & \\
$\begin{array}{l}\text { Hypertension, cardiac } \\
\text { insufficiency }\end{array}$ & 16 & 18 & 0.833 \\
$\begin{array}{l}\text { Pulmonary } \\
\text { incompetence }\end{array}$ & 7 & 9 & 0.786 \\
$\begin{array}{l}\text { Diabetes mellitus } \\
\text { Cerebrovascular } \\
\text { function sequelae }\end{array}$ & 8 & 7 & 1.000 \\
\hline
\end{tabular}

Continuous data were presented as mean \pm SD. TUSP, transurethral split of the prostate; TURP, transurethral resection of the prostate; $\mathrm{Q}_{\max }$ maximum urinary flow rate; PVR, post-void residual; IPSS, international prostate symptoms score; QoL, quality of life.

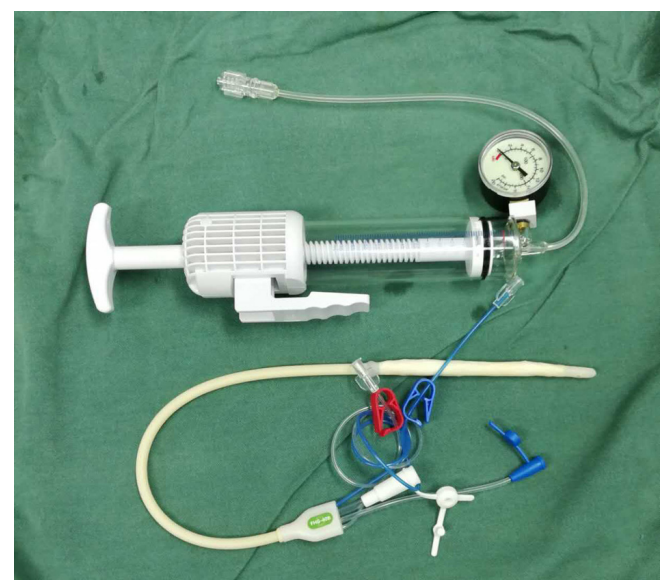

Figure 2 Structure of the double-columnar balloon catheter.

anatomy marker for catheter positioning before inflating. Normal saline inflated the distal balloon to $0.3 \mathrm{MPa}$ and which was closed to the membranous urethra to fix the catheter. The proximal balloon was inflated to $0.3 \mathrm{MPa}$ to expand the prostatic capsule. The cystoscopy appearances before and after the TUSP procedures were shown in Video 1 (before TUSP) and Video 2 (after TUSP).

TURP was performed from the bladder neck to the verumontanum, followed by resection of the anterior lobe, lateral lobes, and the apical tissue until reaching the surgical capsule by skilled surgeons.

\section{Outcomes and follow-up}

The operative time, hemoglobin decrease, catheterization time, postoperative hospital stays, and the incidence of transurethral resection of the prostate syndrome (TURS) were recorded for all patients after the surgery. The primary endpoint for this study was to evaluate the incidence rate of BNC after the surgery. Each patient was followed up for the measurement of IPSS, QoL score, $\mathrm{Q}_{\max }$, and PVR at six months, one year and two years after the first procedure.

\section{Statistical analysis}

The estimated incidence of BNC was $1 \%$ for TUSP and $15 \%$ for TURP according to our earlier outcomes. Thus, 50 patients were needed for each group with an alpha risk of $5 \%$ and a test power of $90 \%$. Data are expressed as mean \pm standard deviation (SD). The student's $t$-test performed statistical analysis or a chi-square test. The Statistical Package for the Social Sciences version 22.0 (SPSS, Chicago, IL, USA) was used for statistical analysis. A P value of $<0.05$ was considered statistically significant.

\section{Results}

\section{The patients' baseline characteristics of both groups}

The patients' baseline characteristics of the TUSP group and TURP group were summarized in Table 1. No significant differences were detected for the baseline characteristics (including age, prostate volume, IPSS, QoL score, $\mathrm{Q}_{\max }$, PVR, PSA level, and concurrent diseases including hypertension, cardiac insufficiency, pulmonary incompetence, diabetes mellitus, acute retention of urine, and cerebrovascular function sequelae) between the two groups $(\mathrm{P}>0.05)$.

\section{The perioperative data of both groups}

Perioperative data were shown in Table 2. The operative time and hemoglobin decrease for the TUSP group are 
Table 2 Intraoperative and postoperative data

\begin{tabular}{lccc}
\hline Parameters & TUSP $(\mathrm{n}=50)$ & TURP $(\mathrm{n}=51)$ & $\mathrm{P}$ value \\
\hline Operative time (mins) & $25.3 \pm 8.6$ & $38.3 \pm 9.3$ & 0.001 \\
$\begin{array}{l}\text { Hemoglobin decrease } \\
(\mathrm{g} / \mathrm{dL})\end{array}$ & $0.31 \pm 0.21$ & $1.43 \pm 0.32$ & 0.001 \\
Catheterization time $(\mathrm{h})$ & $78.9 \pm 16.5$ & $75.9 \pm 15.8$ & 0.352 \\
$\begin{array}{l}\text { Postoperative hospital } \\
\text { stay }(\mathrm{d})\end{array}$ & $1.9 \pm 0.9$ & $2.1 \pm 1.1$ & 0.320 \\
TURS $(\mathrm{n})$ & 0 & & \\
& & 1 & 1.000 \\
\hline
\end{tabular}

Continuous data were presented as mean \pm SD. TUSP, transurethral split of the prostate; TURP, transurethral resection of the prostate; TURS, transurethral resection syndrome.

Table 3 Early and late complications

\begin{tabular}{lccc}
\hline Parameters & TUSP $(\mathrm{n}=50)$ & TURP $(\mathrm{n}=51)$ & $\mathrm{P}$ value \\
\hline Early complications & 2 & 3 & 1.000 \\
Postoperative bleeding & 12 & 16 & 0.506 \\
Micturition frequency & 9 & 11 & 0.804 \\
Micturition urgency & 7 & 6 & 0.775 \\
Micturition pain & 4 & 5 & 1.000 \\
Urinary tract infection & 2 & 4 & 0.678 \\
Recatheterization & 6 & 2 & 0.160 \\
Transient incontinence & & & \\
Late complications & 1 & 8 & 0.031 \\
BNC (1 year) & 0 & 0 & - \\
$\begin{array}{l}\text { Continuous incontinence } \\
\text { (1 year) }\end{array}$ & & &
\end{tabular}

Continuous data were presented as mean \pm SD. TUSP, transurethral split of the prostate; TURP, transurethral resection of the prostate; BNC, bladder neck contracture.

significantly lower than the TURP group $(\mathrm{P}<0.05)$. No significant differences were observed between two groups for catheterization time, postoperative hospital stay, and the incidence of TURS $(\mathrm{P}>0.05)$. The resection weight of prostate tissue for the TURP group is $15.3 \pm 2.8 \mathrm{~g}$. These data showed that TUSP surgery generated short operative time and less blood loss.

\section{The early and late complications of both groups}

Table 3 showed that the early and late complications after surgery, which indicated the BNC incidence in the TUSP group were significantly lower than the TURP group $(\mathrm{P}<0.05)$. No significant differences were observed between two groups for the complications, including postoperative bleeding, micturition urgency, micturition frequency, micturition pain, urinary tract infection, recatheterization, transient incontinence, and continuous incontinence.

\section{The functional results of both groups during follow-up}

Follow-up was done at six months, one year, and two years after the surgical treatment. The IPSS, QoL score, $\mathrm{Q}_{\max }$, and PVR were recorded for each follow-up. As shown in Table 4, no significant differences were detected for the baseline IPSS, QoL score, $\mathrm{Q}_{\max }$, and PVR between the two groups. The IPSS scores of the TUSP group at six months, one year, and two years follow-up were significantly lower than the TURP group. Also, the $\mathrm{Q}_{\max }$ of the TUSP group at six months, one year, and two years follow-up were significantly higher than the TURP group. Further, the QoL score and PVR present no significant differences between the two groups for all the follow-ups. These results showed that TUSP generated better longtime clinical efficacy compared to TURP for small-volume BPH.

\section{Discussion}

In the past decades, TURP was regarded as the golden standard to treat BPH. However, TURP is associated with low efficiency to alleviate the LUTS caused by smallvolume $\mathrm{BPH}$ (12), and with a significantly higher risk of BNC (6). BNC is partially due to the thermal injury followed by tissue resection (5). TUSP involves expanding the prostatic urethra and bladder neck without tissue resection, and thus without thermal injury. In this study, we firstly reported TUSP might be an efficient and safe treatment for small-volume $\mathrm{BPH}(<30 \mathrm{~mL})$, with a lower incidence of postoperative BNC and better longtime clinical outcomes compared to TURP. This study showed that TUSP could be an ideal treatment choice for small-volume BPH $(<30 \mathrm{~mL})$.

Dysuria after surgery may impair a BPH patient's life quality and satisfaction, sometimes even leading to reoperation. The most common cause of postoperative dysuria is $\mathrm{BNC}$; the incidence rate of $\mathrm{BNC}$ ranges from $2.2 \%$ to $9.8 \%$ with different studies $(13,14)$. In this study, the incidence for BNC was $15.7 \%$ for the TURP group, which was higher than those patients with large size BPH. 
Table 4 Functional results during follow-up

\begin{tabular}{|c|c|c|c|c|}
\hline Parameters & Preoperative & 6 months & 1 year & 2 years \\
\hline TUSP & 50 & 50 & 48 & 45 \\
\hline TURP & 51 & 51 & 49 & 45 \\
\hline \multicolumn{5}{|l|}{ IPSS } \\
\hline TURP & $23.9 \pm 2.1$ & $8.9 \pm 2.6$ & $8.2 \pm 1.9$ & $7.6 \pm 1.8$ \\
\hline$P$ value & 0.495 & 0.026 & 0.025 & 0.028 \\
\hline \multicolumn{5}{|l|}{ QoL } \\
\hline TUSP & $4.3 \pm 0.4$ & $2.3 \pm 0.3$ & $2.1 \pm 0.3$ & $2.3 \pm 0.4$ \\
\hline \multicolumn{5}{|l|}{$\mathrm{Q}_{\max }(\mathrm{mL} / \mathrm{s})$} \\
\hline TUSP & $7.8 \pm 1.8$ & $23.5 \pm 6.3$ & $20.7 \pm 5.5$ & $20.8 \pm 5.3$ \\
\hline TURP & $7.6 \pm 1.5$ & $20.8 \pm 6.2$ & $18.5 \pm 4.9$ & $17.3 \pm 5.8$ \\
\hline$P$ value & 0.545 & 0.032 & 0.040 & 0.003 \\
\hline \multicolumn{5}{|l|}{ PVR (mL) } \\
\hline TUSP & $50.3 \pm 27.9$ & $5.8 \pm 3.4$ & $8.2 \pm 5.6$ & $9.7 \pm 6.9$ \\
\hline TURP & $48.5 \pm 21.3$ & $6.2 \pm 5.1$ & $7.1 \pm 6.3$ & $10.1 \pm 8.3$ \\
\hline$P$ value & 0.716 & 0.644 & 0.366 & 0.804 \\
\hline
\end{tabular}

Continuous data were presented as mean \pm SD. TUSP, transurethral split of the prostate; TURP, transurethral resection of the prostate; IPSS, international prostate symptoms; QOL, quality of life; Qmax, maximum urinary flow rate; PVR, post-void residual.

Our results suggested that small-volume BPH patients present a higher risk of BNC after TURP (6). For the small-volume BPH, prostate fibrosis and smooth muscle contractility rather than proliferation or enlargement are usually the main factors contributing to LUTS. Prostate fibrosis is usually associated with chronic prostatitis. For the small-volume BPH with prostate fibrosis and chronic prostatitis, due to thermal injury caused by TURP, resection of the prostate tissue may lead to a new fibrotic pathologic process, which is highly related to BNC (15).

A previous study indicated that TUSP was a safe and long-term efficient treatment for BPH, with minimal invasion, short operative time, and few postoperative complications (4). In this study, the operative time and hemoglobin decrease in the TUSP group were significantly lower than the TURP group. Compared to TUSP, we found that the incidence rate of transient incontinence and catheterization time postoperatively are lower in the TURP group, but the differences are not statistically significant $(\mathrm{P}>0.05)$. For late complications, the incidence of BNC in the TUSP group was significantly lower than the TURP group. No significant differences were observed between the two groups for the other complications. Our data further confirmed the clinical safety and efficacy of TUSP.

TUIP is the first line/standard treatment for the small volume prostates without a middle lobe, according to the EAU 2019 guideline. From the guideline, TURP is also recommended as a treatment choice for the small-volume $\mathrm{BPH}(<30 \mathrm{~mL})$. In our clinical practice, we found some small-volume $\mathrm{BPH}(<30 \mathrm{~mL})$ patients show the hyperplasia of the middle lobe, TUIP is not recommended for these patients with hyperplasia of the middle lobe. This study is a prospective controlled study. We do not want to change the operation choice after the patient's enrollment. Thus, we chose TURP as the control treatment in this study since it may be a reasonable treatment option for all small- 
volume $\mathrm{BPH}(<30 \mathrm{~mL})$ patients, no matter with or without a middle lobe.

For both TURP and TUSP groups of patients, a 2-year follow-up was done. A two-year follow-up was finished for about $90 \%$ of patients. Our data indicated that the IPSS of the TUSP group at six months, one year, and two years follow-up were significantly lower than the TURP group and the $\mathrm{Q}_{\max }$ of the TUSP group at 6 months, 1 year and 2 years follow-up, were significantly higher than the TURP group. These convinced data showed that TUSP generated better longtime clinical efficacy compared to TURP for small-volume BPH.

HoLEP and greenlight laser vaporization of the prostate are the novel techniques for treating $\mathrm{BPH}$. However, their clinical efficacy for patients with small-volume $\mathrm{BPH}$ is still needed to explore (16). The EAU 2019 guideline recommend HoLEP as to the first-line therapeutic choice for large-size $\mathrm{BPH}(>80 \mathrm{~mL})$ and a choice for moderate size BPH $(30-80 \mathrm{~mL})$. However, HoLEP is not recommended for the small-volume BPH $(<30 \mathrm{~mL})$ since the capsule of the prostate is not clear for the HoLEP procedure. For the small-volume BPH $(<30 \mathrm{~mL})$, prostate fibrosis, and smooth muscle contractility rather than proliferation or enlargement are usually the main factors contributing to LUTS (11). The prostate and bladder neck fibrosis could obstruct the bladder outlet. The laser may generate thermal injury and lead to a new fibrotic pathologic process, which increases the risk of BNC after treatment. Thus, we believe TUSP, serving as an expansion surgery without tissue resection and thermal injury might be a better choice for the small-volume BPH.

There were still several limitations to this study. Firstly, it was a single-center study. Secondly, the follow-up period was short. Therefore, some multi-center prospective studies with a longer time follow-up were needed in the future.

\section{Conclusions}

In summary, this study implies that TUSP is an efficient and safe treatment for patients with small-volume $\mathrm{BPH}$ $(<30 \mathrm{~mL})$ with a lower incidence of BNC and better longtime clinical outcomes compared to TURP. It suggested that TUSP could be an ideal treatment choice for small-volume BPH.

\section{Acknowledgments}

Funding: This work was supported by grants from the
National Natural Science Foundation of China (No. 81871153 to JW and No. 81970601 to BW) and the Natural Science Foundation of Zhejiang Province (No. LY18H040007 to JW).

\section{Footnote}

Reporting Checklist: The authors have completed the CONSORT reporting checklist. Available at http://dx.doi. org/10.21037/atm-20-5462

Data Sharing Statement: Available at http://dx.doi. org/10.21037/atm-20-5462

Conflicts of Interest: All authors have completed the ICMJE uniform disclosure form (http://dx.doi.org/10.21037/atm20-5462). The other authors have no conflicts of interest to declare.

Ethical Statement: The authors are accountable for all aspects of the work in ensuring that questions related to the accuracy or integrity of any part of the work are appropriately investigated and resolved. All procedures performed in this study involving human participants were in accordance with the Declaration of Helsinki (as revised in 2013). Given the prospective controlled study design, a written informed consent was obtained from all the patients. This study has been approved by the Ethics Committee of the Second Affiliated Hospital, Zhejiang University School of Medicine (ID of the approval: R2020-525), and was registered in the Clinical Trial Registry of China with the trial number ChiCTR1900021803.

Open Access Statement: This is an Open Access article distributed in accordance with the Creative Commons Attribution-NonCommercial-NoDerivs 4.0 International License (CC BY-NC-ND 4.0), which permits the noncommercial replication and distribution of the article with the strict proviso that no changes or edits are made and the original work is properly cited (including links to both the formal publication through the relevant DOI and the license). See: https://creativecommons.org/licenses/by-nc-nd/4.0/.

\section{References}

1. Nickel JC, Gilling P, Tammela TL, et al. Comparison of dutasteride and finasteride for treating benign prostatic hyperplasia: the Enlarged Prostate International 
Comparator Study (EPICS). BJU Int 2011;108:388-94.

2. Lokeshwar SD, Harper BT, Webb E, et al. Epidemiology and treatment modalities for the management of benign prostatic hyperplasia. Transl Androl Urol 2019;8:529-39.

3. McVary KT, Roehrborn CG, Avins AL, et al. Update on AUA guideline on the management of benign prostatic hyperplasia. J Urol 2011;185:1793-803.

4. Huang W, Huang Z, Xiao G, et al. Effect of transurethral split of the prostate using a double-columnar balloon catheter for benign prostatic hyperplasia. Medicine (Baltimore) 2016;95:e4657.

5. Orihuela E, Pow-Sang M, Motamedi M, et al. Mechanism of healing of the human prostatic urethra following thermal injury Urology. 1996;48:600-8.

6. Tao H, Jiang YY, Jun Q, et al. Analysis of risk factors leading to postoperative urethral stricture and bladder neck contracture following transurethral resection of prostate. INT Braz J Urol 2016;42:302-11.

7. Kang M, Kim M, Choo MS, et al. Urodynamic Features and Significant Predictors of Bladder Outlet Obstruction in Patients With Lower Urinary Tract Symptoms/ Benign Prostatic Hyperplasia and Small Prostate Volume. Urology 2016;89:96-102.

8. Castaneda F, Letourneau JG, Reddy P, et al. Alternative treatment of prostatic urethral obstruction secondary to benign prostatic hypertrophy. Non-surgical balloon catheter prostatic dilatation. Rofo 1987;147:426-9.

9. Huang W, Guo Y, Xiao G, et al. Treatment of benign prostatic hyperplasia using transurethral split of the prostate with a columnar balloon catheter. J Endourol 2015;29:344-50.

Cite this article as: Wang B, Zhang S, Sun C, Du C, He G, Wen J. Comparison between a transurethral prostate split and transurethral prostate resection for benign prostatic hyperplasia treatment in a small prostate volume: a prospective controlled study. Ann Transl Med 2020;8(16):1016. doi: 10.21037/atm-20-5462
10. Kaiho Y, Nakagawa H, Saito H, et al. Nerves at the ventral prostatic capsule contribute to erectile function: initial electrophysiological assessment in humans. Eur Urol 2009;55:148-54.

11. Ma J, Gharaee-Kermani M, Kunju L, et al. Prostatic fibrosis is associated with lower urinary tract symptoms. J Urol 2012;188:1375-81.

12. Li X, Pan JH, Liu QG, et al. Selective transurethral resection of the prostate combined with transurethral incision of the bladder neck for bladder outlet obstruction in patients with small volume benign prostate hyperplasia $(\mathrm{BPH}):$ a prospective randomized study. PLoS One 2013;8:e63227.

13. Michielsen DP, Coomans D. Urethral strictures and bipolar transurethral resection in saline of the prostate: fact or fiction? J Endourol 2010;24:1333-7.

14. Ruszat R, Wyler SF, Seitz M, et al. Comparison of potassium-titanyl-phosphate laser vaporization of the prostate and transurethral resection of the prostate: update of a prospective non-randomized two-centre study. BJU Int 2008;102:1432-8.

15. Maddox M, Pareek G, Al ES, et al. Histopathologic changes after bipolar resection of the prostate: depth of penetration of bipolar thermal injury. J Endourol 2012;26:1367-71.

16. Yin L, Teng J, Huang CJ, et al. Holmium laser enucleation of the prostate versus transurethral resection of the prostate: a systematic review and meta-analysis of randomized controlled trials. J Endourol 2013;27:604-11.

(English Language Editor: J. Chapnick) 